\title{
Deployment of a Web-based Control Laboratory Using HTML5
}

\author{
http://dx.doi.org/10.3991/ijoe.v12i07.5819 \\ Zhongcheng Lei, Wenshan Hu, Hong Zhou \\ Wuhan University, Wuhan, China
}

\begin{abstract}
With the recent achievements in Internet and communication technology and its utilization in online laboratory, remote learning and online laboratory are made possible in the last few decades. This not only provides learning opportunities for people living in rural areas of developing countries, but also offers simple alternatives for those who are already able to access educational resources. The evolution of Web technologies makes it relatively easy to facilitate laboratory deployments, thus, more and more different online laboratory platforms emerge. However, the use of Hyper Text Mark-up Language (HTML5) and related standards such as WebGL and CSS3 in experimental platform, which is future trends and provides full-featured environment, is rare so far. This paper introduces a new HTML5 version of Networked Control System Laboratory (NCSLab), which has been developing for over 10 years since 2006. The 3D version of NCSLab has been developing for almost 5 years in Wuhan University (WHU), whose rendering solution is changed from Flash $3 D$ engine to HTML5 recently. Therefore, Flash player plug-in is eliminated to provide better user experience for users since most of the mainstream web browsers are compatible with HTML5.
\end{abstract}

Index Terms-3D laboratory, HTML5, dual tank, real-time experiment

\section{INTRODUCTION}

In the past few decades, online education [1] has achieved great success. Online courses [2] which is a crucial part of online education are welcomed by learners. Learning platforms, for instance, massive open online courses (MOOC) provide pleasurable opportunities for them to learn with well-known professors from worldrenowned universities. That enhances traditional education with flexibility of learning without limitation on time and location.

However, practical experience, which also plays an important role in the process of learning, especially in engineering education, is often lacked in the form of online education. Thus, theoretical knowledge could not be understood and verified in an easy way. Therefore, online laboratories $[3,4]$ which could work as a supplement of online courses to support online education are proposed by researchers all over the world.

Due to the fact that there are so many technologies and tools for researchers to choose, different kinds of online laboratories with different frameworks using varied solutions are set up by researchers around the world. For example, iLab $[5,6]$ in the USA, WebLabs $[7,8]$ in the UK, VISIR $[9,10]$ in Spain and other kinds of laboratories using MATLAB and Java [11] in other parts of the world.
From a pedagogic perspective, all kinds of laboratories are aimed to provide access for learners to get better educational resources. However, from the technical point of view, learners can get different user experience and obtain different level of knowledge by using these online laboratories.

Hyper Text Mark-up Language 5 (HTML5), whose standard was finalized in 2014 , is adopted by most of the mainstream web browsers now for its full-featured environment. With the use of HTML5 technology, Web-based online laboratories are able to function well with no plugins, which addresses issues such as complicated installation and Web crash caused by plug-ins. Thus, not only online laboratories constructed with other technologies are being changed to HTML5 [12-14], but also new kinds of laboratories are built using HTML5 [15, 16] directly.

The past version of Networked Control System Laboratory (NCSLab) 3D used Flash 3D engine such as Stage3D [17] and Away3D [18, 19] to provide real-time experiment at the expense of Flash player plug-in installation. However, with the rapid development of HTML5 technology, a better solution is available to make the users access the laboratory without the installation of any special software.

In this paper, a new version of NCSLab 3D whose deployment is facilitated with HTML5 is introduced, which provides both virtual and remote experiments with a user friendly interface for better user experience.

The organization of the paper is as follows. Section II introduces the 3D modelling and rendering process, which takes the dual tank as an example. HTML5 is also introduced in this part. Section III presents the real-time experiment of water level control on dual tank test rig. The virtual and remote experiments can both be performed on this single test rig, which can verify each other. The final Section concludes the whole paper.

\section{3D MODELLING AND RENDERING}

In this part, dual tank which is a classic model in control engineering education is demonstrated as an example to illustrate $3 \mathrm{D}$ modelling and rendering. The control purpose of the model is to maintain the water level in the second tank to be at the set point under a certain control algorithm, which can be used to conduct both open-loop and closed-loop control strategies. More importantly, many industrial cases can draw on the experience of the water level control.

The architecture of the NCSLab is demonstrated in details in [17]. It is a multi-tier architecture comprising web browsers, central web server, regional experiment servers, device control units, and experimental devices. Fig. 1 
takes dual tank as an example to describe the system, and it is accessible at http://www.powersim.whu.edu.cn/ ncslab. The picture in Fig. 1 is a photograph of the real dual tank model located in a physical laboratory in Wuhan University (WHU).

The whole model and IP Web camera as well as a Control Unit are included. The dual tank model consists of one water container, two water tank, seven valves, two pumps, two inverter drivers and some water pipes of course, of which the main parts are marked by their names for recognition.

As is shown in Fig. 1, any user such as undergraduate students, graduates, and teachers can use this test rig to conduct experiments as long as the Internet is available. With IP Web camera installed, the dual tank can not only provide hands-on experiments as they used to but can also provide remote experiments.

After the virtual model is constructed and rendered according to the physical model, it can provide another kind of experiment as the virtual experiment. Thus, more users will benefit from it. What' more, the remotely accessibility of the test rig generally expands their functionality with lower expense free from costly room and equipments.

\section{A. 3D Modelling}

To realize the functionality of virtual experiments on a 3D platform, 3D modelling is the initial step should be taken. As every other model which was built before in NCSLab 3D system, dual tank is constructed in a 3D modelling software, which can be seen in Fig. 2.

The models are built on the basis of the real system, in which the color, the size, and the relative position of each part is exactly the same as the real model, thus, users can feel real with the virtual setup while performing experiments.

Inverter drivers are connected to the NetCon Control Unit which is used to execute the algorithm in real-time. Using as a dual tank of water level control with this equipment, one inverter driver is enough with a signal controlling part and a signal acquisition part connected to the Analog/Digital (A/D), Digital/Analog (D/A) part of the Control Unit using wires, respectively.

Fig. 2 shows the entire model of the dual tank in 3DS Max environment. In order to cut down on loading time while carrying out the experiment, the $3 \mathrm{D}$ model should be made to an acceptable size, which means fewer polygons should be contained if possible. In addition, using textures which can be well handled by HTML5 provides an alternative to complicated modelling.

\section{B. From Flash $3 D$ to HTML5}

NCSLab has been built for over ten years since 2006 . NCSLab 3D, which is originated from NCSLab framework has been set up for five years in WHU since 2010. Before HTML5 standard was released, Flash 3D engine is widely used for its strong rendering capability as well as the biggest market share of Adobe Flash. Thus, Flash 3D engine such as Stage3D and Away3D is used to render the models so that they can be used for the Web-based laboratory in the old version of NCSLab 3D.

However, with the development of HTML5 technology, Flash 3D engine is gradually discarded by researchers for its requirement of plug-ins installation. HTML5, which

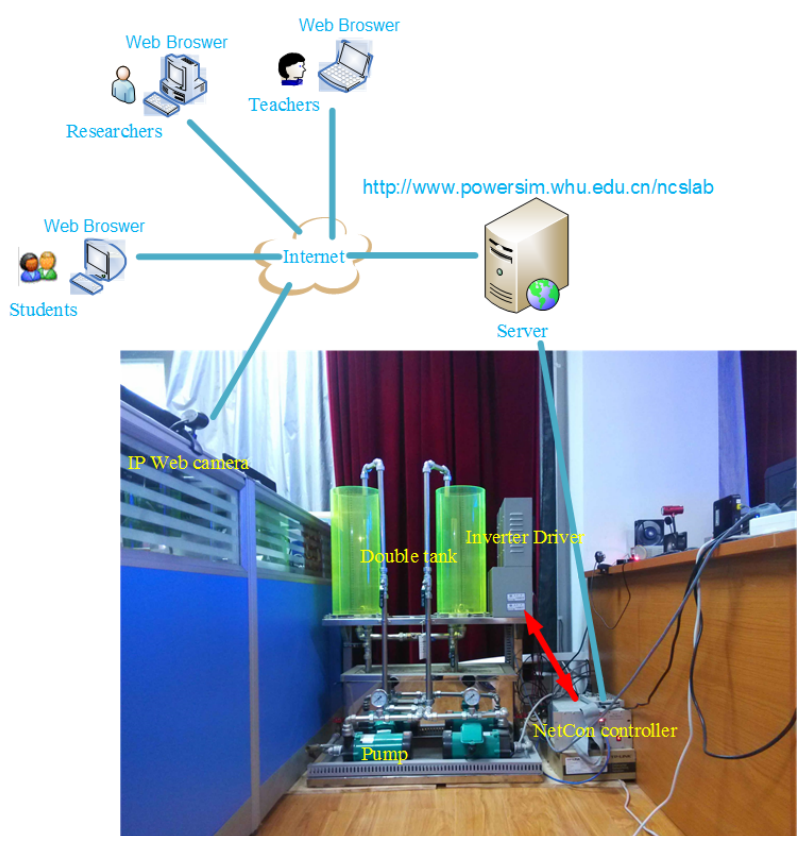

Figure 1. Architecture of the dual tank system

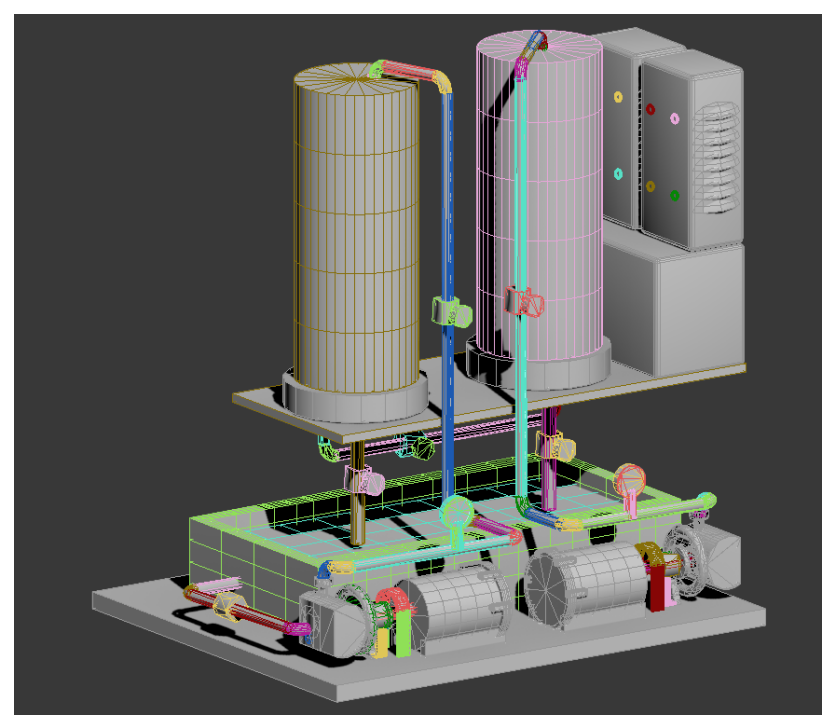

Figure 2. The dual tank model in 3DS Max environment

provides full-featured environment, can offer better user experience without any kind of plug-in. Therefore, the latest HTML5 is adopted to take the place of the Flash 3D engine.

\section{Implementation of HTML5 Interface}

HTML5 is a newly finalized standard, which is full of new features including new elements such as canvas, video, audio. Besides, HTML5 is capable of handling errors in a better way than the old version of HTML.

The proposed Web-based laboratory in this paper mainly uses Three.js under WebGL which is a JavaScript Library to render interactive $3 \mathrm{D}$ computer graphics without use of any plug-in in the web browser.

By using Three.js, 3D models can be easily embedded into the web browser, thus, providing a fluent Web-based $3 \mathrm{D}$ interaction to the users.

The welcome page of NCSLab 3D is depicted as Fig. 3, which is generated by HTML5 technology. It offers an 
entrance to the sub-laboratories like in the real physical laboratories where users can "pick up" test rigs to carry out experiments.

The mainly used JavaScript library is listed as below:

$<$ script src $=$ "threejs/three.min.js" $></$ script $>$

$<$ script

src $=$ "threejs/loaders/DDSLoader.js" $></$ script $>$

$<$ script

src $=$ "threejs/loaders/MTLLoader.js" $></$ script $>$

$<$ script

src $="$ threejs/loaders/OBJMTLLoader.js" $></$ script $>$

in which three.min.js is the basic script to use Three.js. Other scripts such as DDSLoader.js, MTLLoader.js and OBJMTLLoader.js are used for loading textures or materials. HTML5 is capable of dealing with textures better than polygons, which means texture is a better choice when constructing models.

Compared with Flash 3D engine, apart from eliminating Flash player plug-in, HTML5 is easier to render the 3D models and provide a more fluent interface compatible with most of the web browsers.

\section{Communication and Real-Time Animation}

HTML5 technology adopted in this paper makes it possible to provide better user experience. However, it is communication that ensures the experiments in NSCLab $3 \mathrm{D}$ performed in real-time.

The communication has two tasks. One is to establish the synchronization between the widgets (both 3D models and 2D modules) in the web browsers and remote online processes. The other is to pass the users' remote instructions (tuning parameters, changing algorithms etc.) to the Control Unit.

The communication scheme of NCSLab 3D [17] is demonstrated in Fig. 4, in which the data exchange between the web browser and the Control Unit during the experimentation can be seen clearly.

When a user conducts an experiment, a bidirectional data exchange among the web browsers, experiment servers and Control Units is established. If the user starts to monitor the experimental process, the web pages, 3D models, and widgets (2D Modules and 3D Flash Controls) will be downloaded by the web browser from the Experiment Server. With the downloading process completed, the hypertext transfer protocol (HTTP) communication is created automatically between the widgets and the corresponding Data Exchange Module. The data from the RealTime Data Pool are transmitted to the web browser and delivered to the widgets through the HTTP connections.

For each test rig, there is a corresponding Experiment Engine deployed in one of the Experiment Servers. The Experiment Execution Module is the interface to the test rig. When a control algorithm is downloaded and executed in the Control Unit, the transfer control protocol (TCP) communication between the Experiment Execution Module and the Supervisory Control and Data Acquisition (SCADA) Server running in the Control Unit is established automatically. It collects the real-time data from the Control Unit and temporarily stores them into a RealTime Data Pool.

The Real-Time Data Pool, which has a first-in-first-out structure, is a buffer between the web browsers and Con-

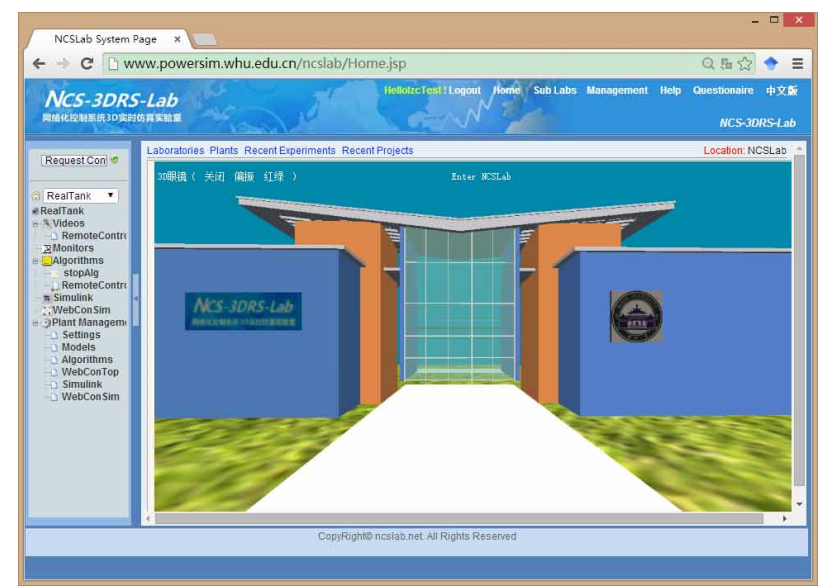

Figure 3. Welcome page of the NCSLab 3D generated by HTML5

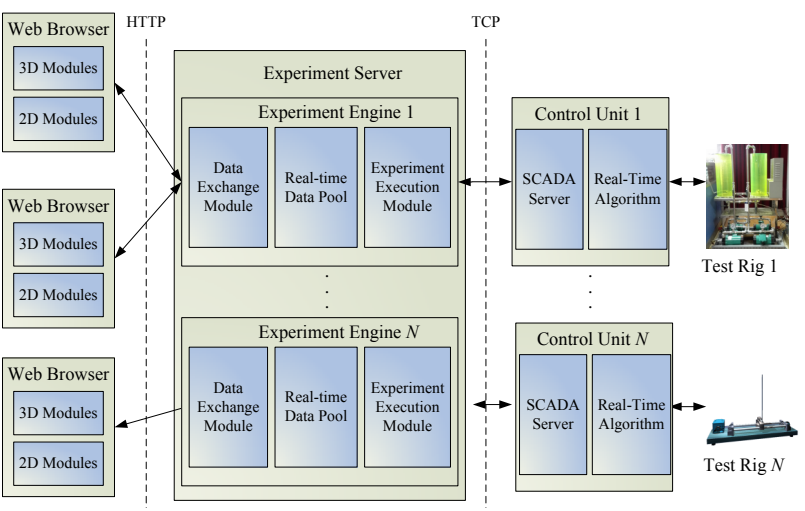

Figure 4. Communication scheme of NCSLab 3D

trol Unit and stores the temporary real-time data in case ofnetwork delay. When the network condition is good (for campus network), the real-time data collected from the Control Unit can be fetched quickly by the web browser. If the network work condition is not perfect (for longdistance Internet transmission), a small time delay is inevitable, but the continuality of the data stream between the web browser and Web Server can still be guaranteed.

To make the virtual $3 \mathrm{D}$ model synchronized with the physical model, data which is retrieved from the Control Unit and is actually numeric should be constructed by animation. For example, water level measured by sensors and actuators is collected by the Control Unit in a numeric form, and eventually is animated by scaling up and down the cylinders inside the two tanks using the numeric data. From the perspective of the user, the water level appears in a $3 \mathrm{D}$ form in the virtual interface and is changed up and down according to the real-time data.

The following is the key codes which are responsible for water animation in the virtual interface of the water level control experiment.

tubes.left.scale. $y=$ leftLevel/70;

tubes.left.position. $y=-(70$-leftLevel $) / 2+40$;

tubes.right.scale. $y=$ rightLevel/70;

tubes.right.position. $y=-(70$-rightLevel $) / 2+40$;

Axis $y$ represents the direction that water supposed to be scaled, while the parameter position is adjusted to make the water in an appropriate position. 


\section{REAL-TIME EXPERIMENT OF DUAL TANK}

In this part, dual tank which was introduced in Section II is selected as a control object to illustrate the control effect of the Web-based laboratory. The virtual interface, which is generated by HTML5 using Three.js of WebGL, is used for virtual experiments, and can be zoomed and viewed from any different angle fluently. While the realtime video interface captured by Web camera is used for remote experiments.

To carry out virtual and remote experiments, the 3D model and the control algorithms are supposed to be combined. The interconnection between the 3D model and control algorithm is shown in Fig. 5, in which all the fitting relations are integrated and the water level corresponding to the virtual and remote experiments can be seen clearly.

The one-one correspondence between the 3D model and the control algorithm make it possible to perform the virtual and remote experiments, which means when a parameter is changed, it is passed down to the NetCon Control Unit and the feedback is sent out to adjust the water level in 3D model and also the real physical model in real-time.

Fig. 6 shows the monitoring interface of water level control experiment on dual tank test rig, in which the upper row is the toolbar including widgets such as number input, curve chart and real-time video. The left widget is the virtual experiment interface, and the middle is the remote experiment interface, while the right one is the real-time curve chart of the water level in the two tank and that of the set point.
As Fig. 6 shows, the water level is changed from $10 \mathrm{~cm}$ to $15 \mathrm{~cm}$, synchronization of water level can be seen in both the virtual interface and real-time video interface.

\section{CONCLUSION AND FUTURE WORKS}

In this paper, a HTML5 version of NCSLab 3D is presented. To demonstrate the new features of NCSLab 3D, The water level control experiment of the dual tank is chosen as a case study. Using the proposed test rig, users can not only carry out remote experiments supported by Web cameras with a strong sense of presence, but also can perform virtual experiments with fluent 3D interaction realized by the latest HTML5 technology. Thus, the two kinds of results can be compared, and they can verify each other.

As most of the mainstream web browsers are compatible with HTML5 now, users can get a good experience of experimentation free from plug-ins. Since HTML5 is good at dealing with textures and rendering, 3D modeling and rendering process can be handled in a relatively easy way.

In the future, there are still works to be done for the improvement of the system. On one hand, the camera in the remote experiment interface can be configured to be controllable at the user side, which can help the user to know about the overall of the test rig.

On the other hand, the virtual experiment interface can also be improved. For example, the water in the virtual experiment interface should be made to look real to provide a better virtual experiment, which is already realized in Flash 3D engine version but remains an issue since HTML5 technology is newly adopted in NCSLab.

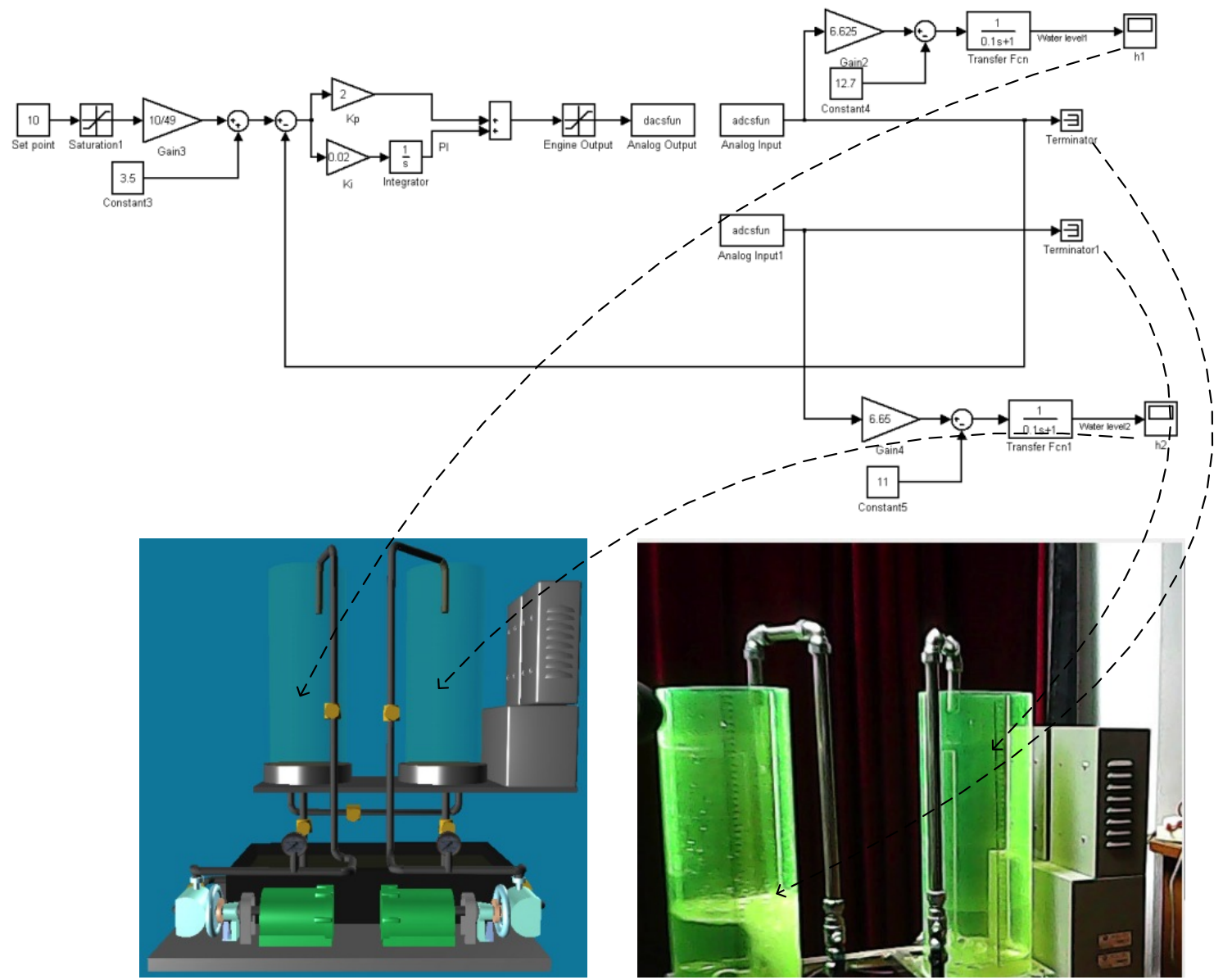

Figure 5. The interconnection between the $3 \mathrm{D}$ virtual/real model and the control algorithm for dual tank test rig 


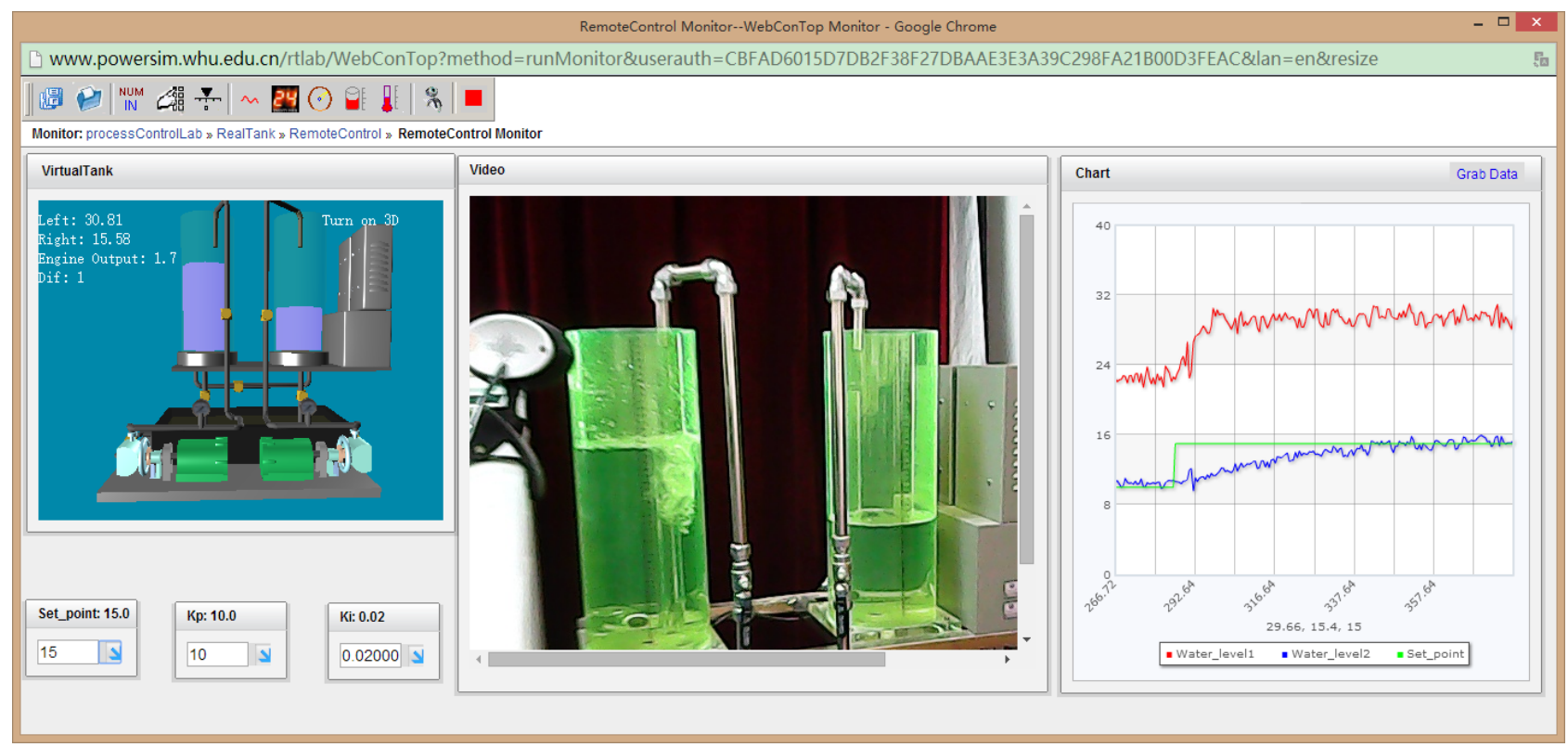

Figure 6. Real-time experiment of water level control on dual tank rig

\section{REFERENCES}

[1] L. Harasim, "Online education," Computer networking and scholarly communication in the twenty-first-century university, pp. 203-214, 1996.

[2] M. H. Schneps, A. Griswold, N. Finkelstein, M. McLeod, and D. P. Schrag, "Using Video to Build Learning Contexts Online," Science, vol. 328, pp. 1119-1120, 2010. http://dx.doi.org/10.1126/ science. 1186934

[3] T. de Jong, S. Sotiriou and D. Gillet, "Innovations in STEM education: the Go-Lab federation of online labs," Smart Learning Environments, vol. 1, pp. 1-16, 2014. http://dx.doi.org/10.1186/ s40561-014-0003-6

[4] M. Ozvoldova and P. Ondrušek, "Integration of Online Labs into Educational Systems," International Journal of Online Engineering (iJOE), vol. 11, pp. 54-59, 2015. http://dx.doi.org/10.3991/ijoe. v11i6.5145

[5] V. J. Harward, J. A. Del Alamo, S. R. Lerman, P. H. Bailey, J. Carpenter, K. DeLong, C. Felknor, J. Hardison, B. Harrison, I. Jabbour, P. D. Long, M. Tingting, L. Naamani, J. Northridge, M. Schulz, D. Talavera, C. Varadharajan, W. Shaomin, K. Yehia, Z. Rabih, and D. Zych, "The iLab Shared Architecture: A Web Services Infrastructure to Build Communities of Internet Accessible Laboratories," Proceedings of the IEEE, vol. 96, pp. 931-950, 2008. http://dx.doi.org/10.1109/JPROC.2008.921607

[6] K. P. Ayodele, I. A. Inyang and L. O. Kehinde, "An iLab for Teaching Advanced Logic Concepts With Hardware Descriptive Languages," Education, IEEE Transactions on, vol. 58, pp. 262268, 2015. http://dx.doi.org/10.1109/TE.2015.2395996

[7] T. Richter, R. Watson, S. Kassavetis, M. Kraft, P. Grube, D. Boehringer, P. de Vries, E. Hatzikraniotis, and S. Logothetidis, "The WebLabs of the University of Cambridge: A study of securing remote instrumentation," in Remote Engineering and Virtual Instrumentation (REV), 2012 9th International Conference on, 2012, pp. 1-5. http://dx.doi.org/10.1109/rev.2012.6293099

[8] K. Bauer and L. A. Mendes, "Weblab of a control experiment in a newborn baby incubator," in Remote Engineering and Virtual Instrumentation (REV), 2015 12th International Conference on, 2015, pp. 163-171. http://dx.doi.org/10.1109/rev.2015.7087285

[9] M. Tawfik, E. Sancristobal, S. Martin, R. Gil, G. Diaz, A. Colmenar, J. Peire, M. Castro, K. Nilsson, J. Zackrisson, X. E. H, L. Kansson, and I. Gustavsson, "Virtual Instrument Systems in Reality (VISIR) for Remote Wiring and Measurement of Electronic Circuits on Breadboard," Learning Technologies, IEEE Transactions on, vol. 6, pp. 60-72, 2013.

[10] M. Tawfik, S. Monteso, F. Garcia-Loro, P. Losada, J. AntonioBarba, E. Ruiz, E. Sancristobal, G. Diaz, J. Peire, and M. Castro,
"Online Experiments With DC/DC Converters Using the VISIR Remote Laboratory," Tecnologias del Aprendizaje, IEEE Revista Iberoamericana de, vol. 10, pp. 310-318, 2015. http://dx.doi.org/ 10.1109/RITA.2015.2486459

[11] G. Farias, R. De Keyser, S. Dormido, and F. Esquembre, "Developing Networked Control Labs: A Matlab and Easy Java Simulations Approach," Industrial Electronics, IEEE Transactions on, vol. 57, pp. 3266-3275, 2010. http://dx.doi.org/10.1109/TIE.2010. $\underline{2041130}$

[12] D. A. H. Samuelsen, J. Bjork and O. H. Graven, "Converting a remote laboratory back end from remote panels in LabVIEW to HTML5," in Remote Engineering and Virtual Instrumentation (REV), 2015 12th International Conference on, 2015, pp. 220-222. http://dx.doi.org/10.1109/rev.2015.7087295

[13] M. Stribny and P. Smutny, "Using HTML5 web interface for visualization and control system," in Carpathian Control Conference (ICCC), 2013 14th International, 2013, pp. 363-366. http://dx.doi.org/10.1109/CarpathianCC.2013.6560570

[14] I. Titov and E. Titov, " Labicom.net - Putting Online LabVIEW Based Remote Laboratory in Less than Four Minutes with WebPager Tool," International Journal of Online Engineering (iJOE), vol. 9, pp. 30-34, 2013. http://dx.doi.org/10.3991/ijoe. v9iS8.3374

[15] D. A. H. Samuelsen, J. Bjork and O. H. Graven, "Work in progress: Simple software solution for accessing remote lab on mobile devices," in Teaching, Assessment and Learning (TALE), 2014 International Conference on, 2014, pp. 69-72. http://dx.doi.org/10.1109/tale.2014.7062588

[16] S. Chen, J. Zornig, N. Chester, P. A. Tregloan, A. Cody, and M. J. Cheesman, "VLPC: A HTML5 pharmacology virtual laboratory," in Remote Engineering and Virtual Instrumentation (REV), 2012 9th International Conference on, 2012, pp. 1-4. http://dx.doi.org/10.1109/rev.2012.6293114

[17] W. Hu, G. P. Liu and H. Zhou, "Web-Based 3-D Control Laboratory for Remote Real-Time Experimentation," Industrial Electronics, IEEE Transactions on, vol. 60, pp. 4673-4682, 2013. http://dx.doi.org/10.1109/TIE.2012.2208440

[18] W. Hu, H. Zhou, Z. Liu, and L. Zhong, "Web-based 3D Interactive Virtual Control Laboratory Based on NCSLab Framework," International Journal of Online Engineering (iJOE), vol. 10, pp. 10-18, 2014. http://dx.doi.org/10.3991/ijoe.v10i6.3845

[19] Z. Lei, W. Hu, H. Zhou, L. Zhong, and X. Gao, "A DC Motor Position Control System in a 3D Real-Time Virtual Laboratory Environment Based on NCSLab 3D," International Journal of Online Engineering (iJOE), vol. 11, pp. 49-55, 2015. http://dx.doi.org/10.3991/ijoe.v11i3.4556 


\section{AUTHORS}

Zhongcheng Lei is with Department of Automation, School of Power and Mechanical Engineering, Wuhan University, 430072, China. (zhongcheng.lei@whu.edu.cn)

Wenshan Hu is with Department of Automation, School of Power and Mechanical Engineering, Wuhan University, 430072, China. (wenshan.hu@whu.edu.cn).

Hong Zhou is with Department of Automation, School of Power and Mechanical Engineering, Wuhan University, 430072, China. (hzhouwuhee@whu.edu.cn).

This article is an extended and modified version of a paper presented at the International Conference on Remote Engineering \& Virtual Instrumentation (REV2016), held at UNED, Madrid, Spain, February 24-26, 2016. The work was supported by the National Science Foundation of China under Grant 61374064. Submitted 15 May 2016. Published as resubmitted by the authors 12 June 2016 . 